\section{ARTIGO DE REVISÃO REVIEW ARTICLE}

Palavras-chave:

câncer de colo do útero, políticas públicas, Brasil, saúde da mulher.

\title{
O câncer de colo do útero no Brasil: uma retrospectiva sobre as políticas públicas voltadas à saúde da mulher
}

\author{
Cervical cancer in Brazil: a retrospective on \\ public policies for women's health \\ Carolina Terumi Tsuchiya', Tatiana Lawrence', Mariana Stutz Klen², \\ Roberta Arinelli Fernandes², Marcia Regina Alves ${ }^{1}$ \\ DOI: 10.21115/JBES.v9.n1.p137-47
}

\begin{abstract}
RESUMO
O câncer de colo do útero é a terceira neoplasia mais incidente entre as brasileiras, com taxa de mortalidade acima de 5/100 mil mulheres, apesar de possuir bom prognóstico quando diagnosticado em fases precoces. No Brasil, políticas públicas voltadas para o câncer de colo do útero vêm sendo desenvolvidas desde a década de 1970 e incluíram diversos programas de rastreamento, que conseguiram aumentar o acesso ao teste de Papanicolau, com patamar de cobertura estável nos últimos anos, em torno dos 83\%. Em 2014, a vacina anti-HPV foi incluída no Calendário Nacional de Vacinação. O mais recente Plano de Ações Estratégicas possui metas de aumento da cobertura de exame citopatológico e tratamento de todas as mulheres com lesões precursoras. Outras duas iniciativas foram criadas: o Programa de Qualificação de Ginecologistas para Assistência Secundária às Mulheres com Alterações Citológicas Relacionadas às Lesões Intraepiteliais e ao Câncer de Colo do Útero e a formalização da Rede Colaborativa para a Prevenção do Câncer de Colo do útero. Mesmo com o constante avanço dessas medidas, mais de $70 \%$ das brasileiras são diagnosticadas em fases avançadas da doença, o que impacta negativamente no prognóstico. A análise dos protocolos de tratamento voltados para a saúde coletiva mostra defasagem em relação ao cenário internacional e nacional preconizado por sociedades médicas, especialmente no tratamento de fases tardias da doença. Apesar dos avanços na difusão de medidas preventivas e alcance de ampla cobertura do rastreamento, o câncer de colo do útero continua a ser um problema de saúde importante no país.
\end{abstract}

\section{Keywords:}

uterine cervical neoplasm, public policies, Brazil, women's health.

\begin{abstract}
Cervical cancer is the third most frequent neoplasm among Brazilian women, with a mortality rate of more than 5/100 thousand women, despite having a good prognosis when diagnosed in the early stages. In Brazil, public policies for cervical cancer have been developed since the 1970s and have included several screening programs that have been able to increase access to the Papanicolaou test, with a stable coverage level in recent years, around 83\%. In 2014, the anti-HPV vaccine was included in the National Vaccination Calendar. The most recent Strategic Action Plan has targets to increase coverage of cytopathological examination and treatment of all women with precursor lesions. Two other initiatives were created: the Qualification Program of Gynecologists for Secondary Care for Women with Cytologic Alterations Related to Intraepithelial Lesions and Cervical Cancer, and the formalization of the Collaborative Network for the Prevention of Cervical Cancer. Even with constant progress of these measures, more than $70 \%$ of Brazilian women are diagnosed in advanced stages of the disease, which negatively impacts the prognosis. The analysis of treatment protocols aimed at public health shows lag in relation to international and national scenario recommended by medical societies, especially in the treatment of late stage disease. Despite the advances in the dissemination of preventive measures and the scope of extensive screening coverage, cervical cancer remains a major health problem in the country.
\end{abstract}

Recebido em: 19/01/2017. Aprovado para publicação em: 11/03/2017.

1. F. Hoffmann-La Roche Ltd, São Paulo, SP, Brasil.

2. Sense Company, Rio de Janeiro, RJ, Brasil.

Instituição onde o trabalho foi executado: F. Hoffmann-La Roche Ltd e Sense Company.

Financiamento: $O$ estudo foi financiado pela F. Hoffmann-La Roche Ltd.

Autor correspondente: Carolina T. Tsuchiya. Av. Engenheiro Billings, 1729 - Jaguaré, São Paulo, SP, Brasil.

Telefone: +55 (11) 3719-8063. E-mail: carolina.tsuchiya@roche.com. 


\section{Câncer de colo do útero}

O câncer de colo do útero (CCU) é uma doença de natureza crônica, com origem em alterações intraepiteliais que podem se transformar em um processo invasor [World Health Organization (WHO), 2014]. Pode originar-se do epitélio escamoso da ectocérvice (carcinoma de células escamosas - CCE) ou do epitélio escamoso colunar do canal cervical (adenocarcinoma cervical - ACC). O CCE e o ACC representam 90\% e 10\% dos casos de CCU, respectivamente [Ministério da Saúde (Brasil). Instituto Nacional do Câncer (Inca), 2000].

Segundo a Organização Mundial da Saúde (OMS), uma infecção persistente ou crônica de um ou mais tipos de papilomavírus humano (HPV) é considerada a causa primária do CCU [World Health Organization (WHO), 2014]. O HPV de alto risco é encontrado em 99,7\% dos CCUs (UK National Screening Committee, 2016), sendo a infecção mais comumente adquirida por meio de relações sexuais, geralmente no início da vida sexual. Na maioria dos indivíduos afetados por esse vírus, as infecções são espontaneamente resolvidas. Nos casos em que as infecções se apresentam persistentes, pode haver progressão para o CCU em 10 a 20 anos após a infecção [World Health Organization (WHO), 2014].

As lesões cervicais consideradas precursoras possuem, do ponto de vista cito-histopatológico, diferentes graus evolutivos classificados como neoplasia intraepitelial cervical (NIC) [Ministério da Saúde (Brasil). Instituto Nacional do Câncer (Inca), 2000]. A NIC é apresentada como uma longa fase pré-invasiva da doença e é classificada em graus I, II e III, dependendo da proporção da espessura do epitélio que apresenta células maduras e diferenciadas. Os graus II e III, considerados os mais graves, apresentam maior proporção da espessura do epitélio composto de células indiferencia- das, possuindo maior probabilidade de progressão para o câncer e, assim, sendo considerados seus reais precursores. As NICs de grau I geralmente regridem em períodos entre 12 e 24 meses ou não progridem para graus II ou III, não sendo consideradas lesões precursoras [Ministério da Saúde (Brasil). Instituto Nacional de Câncer (Inca), 2011].

A Tabela 1 apresenta as nomenclaturas citopatológica e histopatológica utilizadas desde o início da realização do exame citopatológico para o diagnóstico das lesões cervicais escamosas e suas equivalências. As nomenclaturas de Papanicolau, que utilizam classes numéricas, e da OMS, que usam o termo displasia, não são recomendadas, pois diferenciam indevidamente graus de doença pré-invasiva [Ministério da Saúde (Brasil). Instituto Nacional de Câncer (Inca), 2011]. A partir da confirmação do diagnóstico por meio da biópsia, a paciente com CCU é estadiada de acordo com o estádio clínico da Federação Internacional de Ginecologia e Obstetrícia (FIGO) (Tabela 2) [Ministério da Saúde (Brasil). Instituto Nacional de Câncer (Inca), 2011].

\section{Epidemiologia do CCU}

De acordo com o Globocan, o CCU é o quarto tipo de câncer mais comum em mulheres, e o sétimo mais comum entre todos os tipos de câncer, com aproximadamente 528 mil novos casos estimados em 2012. Sua incidência é maior em países menos desenvolvidos quando comparada aos países mais desenvolvidos (Figura 1) (Ferlay et al., 2015).

No Brasil, a estimativa para o ano de 2014 publicada pelo Inca, válida também para 2015, apontou o CCU como o terceiro tipo de câncer mais incidente entre as mulheres, sendo 15.590 casos novos por ano, com um risco estimado de 15,33 casos a cada 100 mil mulheres [Ministério da Saúde (Brasil). Instituto Nacional de Câncer José Alencar Gomes da Silva (Inca), 2014].

Tabela 1. Nomenclaturas citopatológica e histopatológica utilizadas desde o início da realização do exame citopatológico para o diagnóstico das lesões cervicais escamosas e suas equivalências - Inca, 2011 [Ministério da Saúde (Brasil). Instituto Nacional de Câncer (Inca), 2011]

\begin{tabular}{llll}
\hline $\begin{array}{l}\text { Classificação } \\
\text { citológica de } \\
\begin{array}{l}\text { Papanicolau } \\
(\mathbf{1 9 4 1 )}\end{array}\end{array}$ & $\begin{array}{l}\text { Classificação histológica da OMS } \\
(\mathbf{1 9 5 2 )}\end{array}$ & $\begin{array}{l}\text { Classificação histológica } \\
\text { de Richart (1967) }\end{array}$ & $\begin{array}{l}\text { Classificação citológica brasileira } \\
\mathbf{( 2 0 0 6 )}\end{array}$ \\
\hline Classe I & - & - & - \\
\hline Classe II & - & - & Alterações benignas \\
\hline- & - & - & Atipias de significado indeterminado \\
\hline Classe III & Displasia leve & NIC I & LSIL \\
\hline Classe IV & Cisplasia moderada e acentuada & NIC II e NIC III & HSIL \\
\hline Classe V & Carcinoma in situ & NIC III & HSIL \\
\hline
\end{tabular}

OMS: Organização Mundial da Saúde; NIC: neoplasia intraepitelial cervical; LSIL: lesão intraepitelial escamosa de baixo grau; HSIL: Iesão intraepitelial escamosa de alto grau; AIS: adenocarcinoma in situ. 
Tabela 2. Estadiamento do CCU de acordo com a FIGO (Pecorelli et al., 2009)

\begin{tabular}{|c|c|}
\hline Estádio I & $\begin{array}{l}\text { O carcinoma se encontra estritamente } \\
\text { confinado à cérvice }\end{array}$ \\
\hline IA & $\begin{array}{l}\text { Carcinoma invasivo que só pode ser diagnosticado } \\
\text { por microscopia, com invasão de maior } \\
\text { profundidade } \leq 5 \mathrm{~mm} \text { e maior extensão } \geq 7 \mathrm{~mm}\end{array}$ \\
\hline$|A|$ & $\begin{array}{l}\text { Medição da invasão estromal } \leq 3,0 \mathrm{~mm} \text { em } \\
\text { profundidade e extensão } \leq 7,0 \mathrm{~mm}\end{array}$ \\
\hline IA2 & $\begin{array}{l}\text { Medição da invasão estromal > 3,0 mm e não > 5,0 } \\
\text { mm com extensão não > 7,0 mm }\end{array}$ \\
\hline IB & $\begin{array}{l}\text { Lesões visíveis clinicamente limitadas à cérvice } \\
\text { uterina ou câncer pré-clínico maiores que o } \\
\text { estágio IA* }\end{array}$ \\
\hline |B1 & $\begin{array}{l}\text { Lesões visíveis clinicamente } \leq 4,0 \mathrm{~cm} \text { em sua maior } \\
\text { dimensão }\end{array}$ \\
\hline IB2 & $\begin{array}{l}\text { Lesões visíveis clinicamente }>4,0 \mathrm{~cm} \text { em sua maior } \\
\text { dimensão }\end{array}$ \\
\hline Estádio II & $\begin{array}{l}\text { Invasão do carcinoma cervical além do útero, mas } \\
\text { sem alcançar a parede pélvica ou o terço inferior } \\
\text { da vagina }\end{array}$ \\
\hline$\| \mathrm{A}$ & Sem invasão parametrial \\
\hline$\| A 1$ & $\begin{array}{l}\text { Lesões visíveis clinicamente } \leq 4,0 \mathrm{~cm} \text { em sua maior } \\
\text { dimensão }\end{array}$ \\
\hline$\| \mathrm{A} 2$ & $\begin{array}{l}\text { Lesões visíveis clinicamente }>4,0 \mathrm{~cm} \text { em sua maior } \\
\text { dimensão }\end{array}$ \\
\hline$\| B$ & Com invasão parametrial óbvia \\
\hline Estádio III & $\begin{array}{l}\text { O tumor se estende à parede pélvica e/ou envolve } \\
\text { o terço inferior da vagina e/ou causa hidronefrose } \\
\text { ou disfunção renal** }\end{array}$ \\
\hline$\| I I A$ & $\begin{array}{l}\text { O tumor envolve o terço inferior da vagina, sem } \\
\text { extensão para a parede pélvica }\end{array}$ \\
\hline$\| I I B$ & $\begin{array}{l}\text { Extensão para a parede pélvica e/ou hidronefrose } \\
\text { ou disfunção renal }\end{array}$ \\
\hline Estádio IV & $\begin{array}{l}\text { O carcinoma apresenta extensão além da pelve } \\
\text { verdadeira ou envolveu (a partir de biópsia } \\
\text { comprovada) a mucosa da bexiga ou do reto. Um } \\
\text { edema bolhoso não permitiria estadiar o caso } \\
\text { como estádio IV }\end{array}$ \\
\hline IVA & Propagação do câncer para os órgãos adjacentes \\
\hline IVB & Propagação do câncer para os órgãos distantes \\
\hline
\end{tabular}

* Todas as lesões macroscopicamente visíveis, mesmo com invasão superficial, foram alocadas para o estágio de carcinoma IB. A invasão está limitada a uma invasão estromal com profundidade máxima de 5,00 mm e extensão horizontal não $>$ 7,00 milímetros. A profundidade da invasão não deve ser $>5,00$ milímetros tomada a partir da base do epitélio do tecido original (seja superficial ou glandular). A profundidade da invasão deve ser sempre indicada em $\mathrm{mm}$, mesmo naqueles casos com "invasão estromal precoce (mínima)" ( 1 mm). O envolvimento de espaços vasculares/linfáticos não deve alterar o estadiamento. ** No exame retal, não há espaço livre de câncer entre o tumor e a parede pélvica. Todos os casos com hidronefrose ou disfunção renal estão incluídos, a menos que sejam conhecidos como sendo devidos a outra causa.

FIGO: Federação Internacional de Ginecologia e Obstetrícia; CCU: câncer de colo do útero.

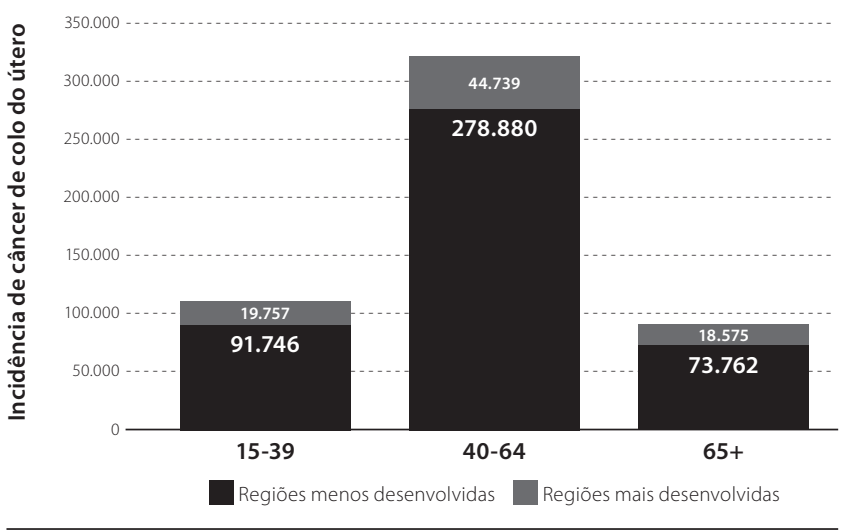

Figura 1. Estimativa para o ano de 2012 da incidência, por grupo etário, de câncer de colo do útero de acordo com o grau de desenvolvimento dos países. Adaptada de: Globocan, 2012 [World Health Organization (WHO), 2012].

Para 2016, foram estimados 16.340 novos casos de CCU no Brasil, o que representa um aumento de 4,8\% em relação a 2014, mantendo-se como o terceiro câncer mais incidente entre as mulheres brasileiras, com taxa de incidência estimada em 15,85 por 100 mil mulheres (Tabela 3) [Ministério da Saúde (Brasil). Instituto Nacional de Câncer José Alencar Gomes da Silva (Inca), 2016].

Entre os tipos de câncer mais comuns em mulheres, exceto os tumores de pele não melanoma, a taxa de incidência do CCU ocupa a primeira posição na região Norte e a segunda nas regiões Centro-Oeste e Nordeste. Nas regiões Sudeste e Sul, o CCU representa o terceiro e o quarto tipo de câncer mais incidente, respectivamente [Ministério da Saúde (Brasil). Instituto Nacional de Câncer José Alencar Gomes da Silva (Inca), 2016]. As taxas de incidência para cada região geográfica do Brasil estão apresentadas na Figura 2.

Tabela 3. Distribuição proporcional dos 10 tipos de câncer mais incidentes estimados para 2016 para mulheres, exceto pele não melanoma. Adaptada de: Ministério da Saúde (Brasil). Instituto Nacional de Câncer José Alencar Gomes da Silva (Inca), 2016.

\begin{tabular}{lcc}
\hline Localização primária & Casos & $\%$ \\
\hline Mama feminina & 57.960 & 28,1 \\
\hline Cólon e reto & 17.620 & 8,6 \\
\hline Colo do útero & 16.340 & 7,9 \\
\hline Traqueia, brônquio e pulmão & 10.890 & 5,3 \\
\hline Estômago & 7.600 & 3,7 \\
\hline Corpo do útero & 6.950 & 3,4 \\
\hline Ovário & 6.150 & 3,0 \\
\hline Glândula tireoide & 5.870 & 2,9 \\
\hline Linfoma não Hodgkin & 5.030 & 2,4 \\
\hline Sistema nervoso central & 4.830 & 2,3 \\
\hline
\end{tabular}




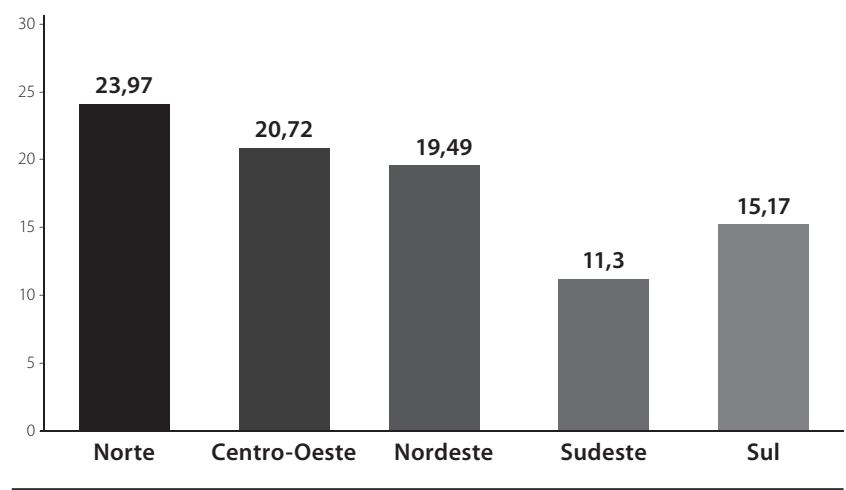

Figura 2. Taxas de incidência por 100 mil habitantes, estimadas para 2016, para o CCU, segundo as regiões geográficas brasileiras. Adaptada de: Ministério da Saúde (Brasil). Instituto Nacional de Câncer José Alencar Gomes da Silva (Inca), 2016.

Em uma análise epidemiológica de 51.842 casos de CCU, realizada por Nogueira-Rodrigues et al. (2014), foi demonstrado que as pacientes com CCE eram mais jovens e, frequentemente, solteiras, negras, sem escolaridade e fumantes. A maioria dessas pacientes era do sudeste brasileiro $(42,1 \%)$. O CCU no estádio I foi o mais frequente nos casos de ACC (33,3\%) e representou $21,3 \%$ dos casos de CCE. A idade média das pacientes foi de $51,2 \pm 14,5$ anos $(51,1 \pm 14,6$ em pacientes com CCE versus 52,1 $\pm 13,8$ em pacientes com ACC; $p<$ $0,001)$. A porcentagem de pacientes com ACC aumentou em 55,9\% (de 10,2\% para 15,9\%) nos últimos 10 anos. Das mulheres avaliadas no estudo, 20\% não apresentaram resposta ao tratamento, culminando em registro de óbito em $12,8 \%$ das pacientes com ACC e 12,6\% das com CCE. Das pacientes com doença em estágio inicial ( $F \mid G O \leq \| A)$, a intervenção cirúrgica foi o único tratamento em $37,4 \%$ e $35,7 \%$ das pacientes com ACC e CCE, respectivamente, e 3,1\% delas tiveram resposta inadequada a essa estratégia de tratamento. Das pacientes em estádio $\mathrm{FIGO} \geq \| \mathrm{IB}$, a radioterapia foi o único tratamento aplicado em $43,9 \%$ das pacientes com ACC e $52 \%$ das portadoras de CCE, e resposta inadequada foi observada em 33,3\% e 31,6\% das pacientes, respectivamente (Nogueira-Rodrigues et al., 2014).

Segundo a OMS, a faixa etária mais afetada pelo CCU se encontra entre 30 e 49 anos de idade [World Health Organization (WHO), 2014]. Os resultados colpocitológicos positivos para CCU são mais frequentes nas mulheres em cenário de vulnerabilidade social. Aproximadamente 500 mil novos casos de CCU são registrados por ano, sendo a maioria diagnosticada em países em desenvolvimento (Nogueira-Rodrigues et al., 2014). Quando as taxas de incidência e mortalidade do Brasil são comparadas ao resto do mundo, os valores tendem a ser intermediários em relação aos países em desenvolvimento e superiores aos países desenvolvidos, que apresentam programas de detecção precoces eficientes [Ministério da Saúde (Brasil). Secretaria de Atenção à Saúde, 2013]. O es- tudo de base populacional que comparou a incidência idade-específica do CCU entre o Brasil e o Reino Unido (Harper \& Vierthaler, 2011) mostrou uma taxa semelhante até os 30 anos, a princípio demonstrando equivalência de exposição aos fatores de risco. Entretanto, após essa idade, ocorre franca separação das curvas, mostrando uma incidência quase quatro vezes maior nas mulheres brasileiras em relação às britânicas aos 65 anos (Figura 3). Essa disparidade pode ser explicada pela eficiência nos métodos de rastreamento e acesso ao tratamento médico adequado pelas mulheres britânicas.

De acordo com o Globocan, a taxa estimada, padronizada por idade, de incidência de câncer por sexo, local do câncer e região, de 2012, para o CCU foi de 14,0 por 100 mil (Tabela 4) na perspectiva mundial, sendo de 9,9 nos países desenvolvidos e 15,7 nos em desenvolvimento (Figura 4). Tal parâmetro apresentou também taxa de 20,4 na América do Sul contra 6,6 na América do Norte e 8,7 no Norte Euro peu (Ferlay et al., 2015).

Em relação à mortalidade, o número estimado de óbitos por CCU foi de aproximadamente 266 mil no mundo em

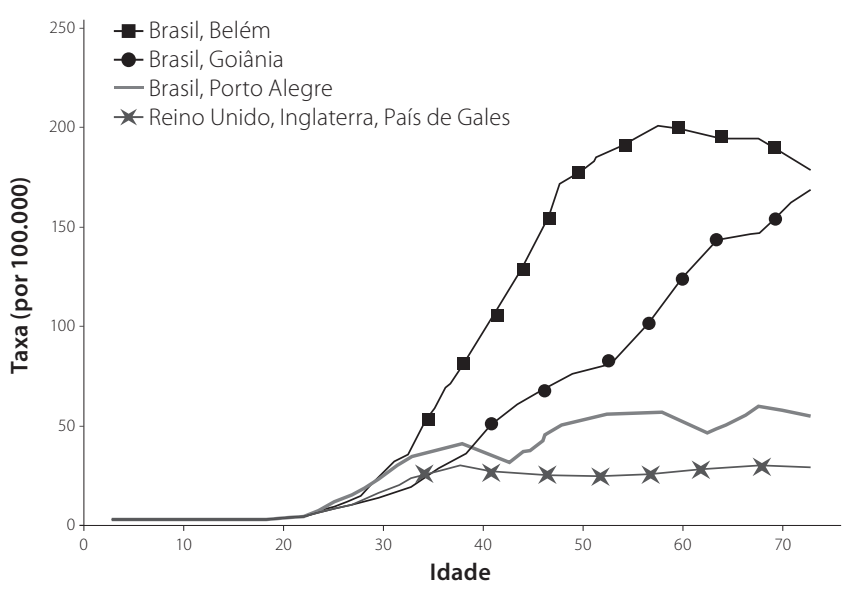

Figura 3. Taxas de incidência idade-específica do câncer de colo do útero comparadas entre Brasil e Reino Unido (Parkin et al., 1997).

Tabela 4. Estimativa mundial de novos casos de CCU e mortes por CCU, taxa padronizada por idade e riscos cumulativos para 75 anos - Globocan, 2012 (Ferlay et al., 2015)

\begin{tabular}{lcc}
\hline CCU & Novos casos & Óbitos \\
\hline Casos (milhares) & 528 & 266 \\
\hline (\%) & 3,7 & 3,2 \\
\hline $\begin{array}{l}\text { Taxa padronizada por } \\
\text { idade/100.000 (mundo) }\end{array}$ & 14,0 & 6,8 \\
\hline Risco cumulativo (\%) (0-74) & 1,4 & 0,8 \\
\hline
\end{tabular}

CCU: câncer de colo do útero. 


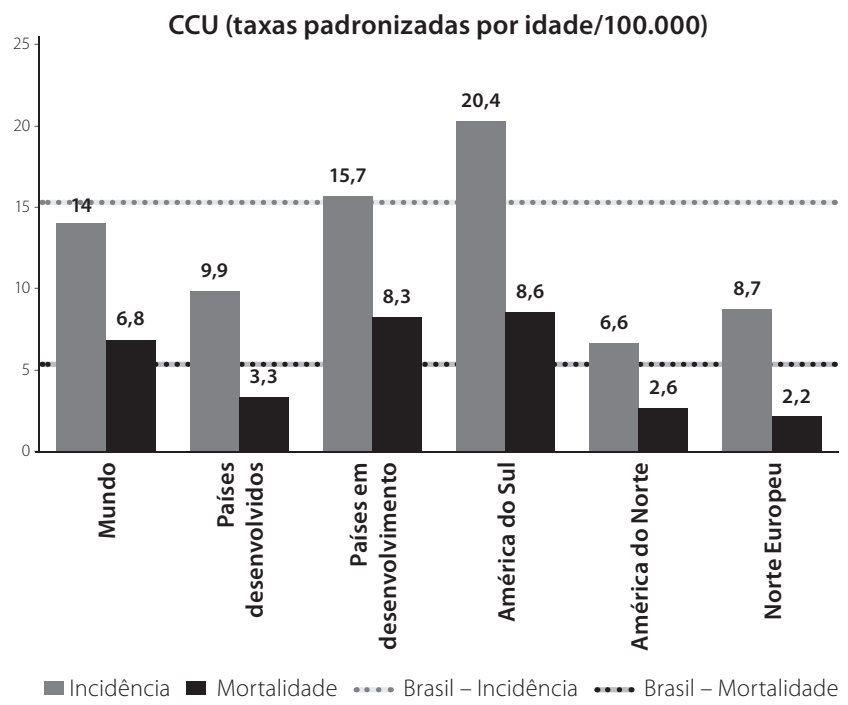

Dados do Brasil retirados da estimativa de 2014 do Inca e do Sistema de Informação sobre Mortalidade de 2013 [Ministério da Saúde (Brasil). Departamento de Informática do SUS (Datasus), 2013; Ministério da Saúde (Brasil). Instituto Nacional de Câncer (Inca), 2011].

CCU: câncer de colo do útero.

Figura 4. Taxas padronizadas por idade de incidência e mortalidade de CCU por região do mundo e no Brasil. Adaptada de: Globocan, 2012 (Ferlay et al., 2015).

2012 (Tabela 4); 87\% desses óbitos ocorreram em países em desenvolvimento (35,5 mil nos países desenvolvidos e 230,2 mil nos em desenvolvimento). No continente americano, o número estimado de mortes foi de 19,4 mil na América do Sul e de 7,1 mil na América do Norte, enquanto o Norte Europeu apresentou 17,8 mil. O risco médio de morte por CCU antes dos 75 anos é três vezes maior nos países em desenvolvimento do que nos países desenvolvidos (Ferlay et al., 2015).

Na Figura 5, podem ser observadas no mapa as taxas de incidência de CCU segmentadas por região brasileira [Ministério da Saúde (Brasil). Instituto Nacional de Câncer José Alencar Gomes da Silva (Inca), 2014] e as taxas de mortalidade [Ministério da Saúde (Brasil). Departamento de Informática do SUS (Datasus), 2013], comparadas a valores de diferentes países [World Health Organization (WHO), 2013; World Health Organization (WHO), 2015].

Em análise evolutiva das últimas três décadas (Figura 6) [Ministério da Saúde (Brasil). Instituto Nacional de Câncer (Inca). Organização Pan-Americana da Saúde, 2014], a taxa de mortalidade por CCU no Brasil se manteve praticamente constante, o que provavelmente se relaciona à manutenção das altas taxas de estadiamento avançado no momento do diagnóstico e à limitação das opções e resultados terapêuticos nessa situação. Variações regionais também são observadas na evolução da mortalidade. Esses dados evidenciam as dificuldades suplementares para o controle da doença no país (Teixeira, 2015).

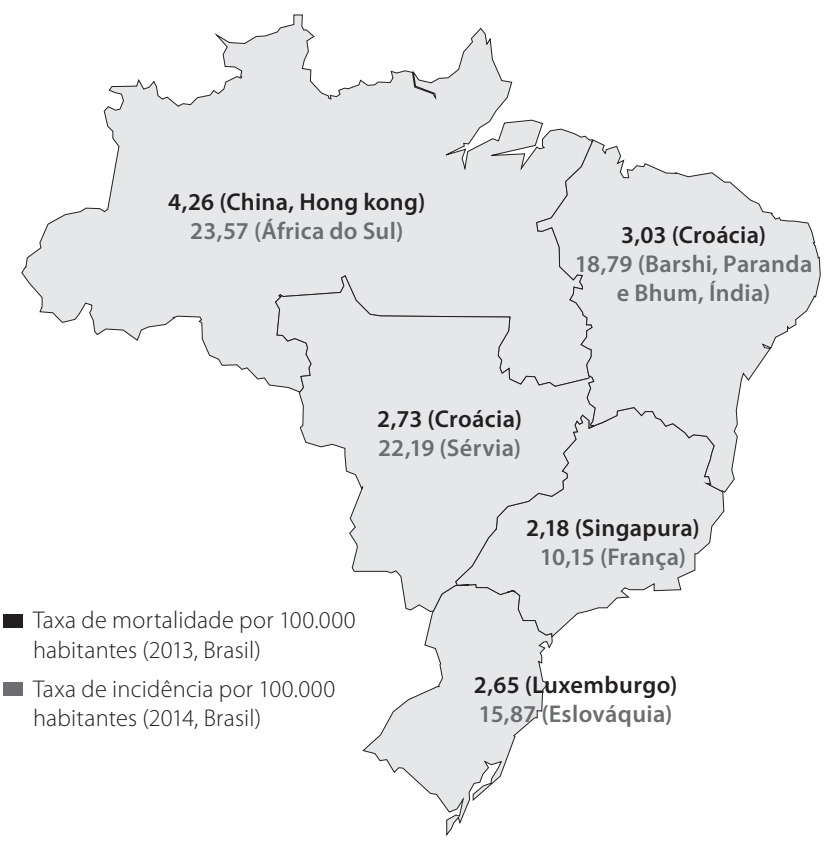

Os países apresentados entre parênteses são aqueles que apresentaram taxas semelhantes às nacionais expostas no mapa. As taxas de incidência internacionais utilizadas para correspondência com as nacionais são referentes aos anos de 20032007. As taxas de mortalidade internacionais utilizadas para correspondência com os dados nacionais apresentados datam de 2013, assim como os valores brasileiros. Valores nacionais para taxa de incidência por 100 mil habitantes foram retirados das estimativas de 2014 do Inca [Ministério da Saúde (Brasil). Instituto Nacional de Câncer (Inca), 2011], e os valores internacionais utilizados para efeito de comparação foram obtidos a partir da base de dados Cl5 (Cancer Incidence in Five Continents) do CANCER Mondial, sendo eles: 23,8 (África do Sul), 18,7 (Barshi, Paranda e Bhum; Índia), 23,2 (Sérvia), 10,1 (França) e 15,8 (Eslováquia), correspondendo às regiões Norte, Nordeste, Centro-Oeste, Sudeste e Sul, respectivamente [World Health Organization (WHO), 2013].

As taxas brasileiras de mortalidade foram obtidas pelo Sistema de Informação sobre Mortalidade [Ministério da Saúde (Brasil). Departamento de Informática do SUS (Datasus), 2013] de 2013, e os valores internacionais de mortalidade foram obtidos na base de dados da OMS Cancer Mortality Database, de 2015, sendo eles: 4,26 (China, Hong Kong), 3,03 (Croácia), 2,18 (Singapura) e 2,65 (Luxemburgo), correspondendo às regiões Norte, Nordeste e Centro-Oeste, Sudeste e Sul, respectivamente [World Health Organization (WHO), 2015].

Figura 5. Correspondência entre os valores nacionais, por região do Brasil, da taxa de incidência (correspondente a 2014) e taxa de mortalidade (por 100 mil habitantes; correspondente a 2013), e as taxas equivalentes de outros países [Ministério da Saúde (Brasil). Instituto Nacional de Câncer (Inca), 2011; World Health Organization (WHO), 2013; World Health Organization (WHO), 2015].

\section{Abordagem da OMS para a paciente com CCU}

A abordagem da OMS para a prevenção e controle do CCU inclui intervenções programáticas ao longo da vida para prevenir a infecção pelo HPV. O objetivo de um programa global de prevenção e controle do CCU é diminuir sua carga por meio da redução de infecções por HPV, da detecção e tratamento de lesões cervicais pré-cancerosas em tempo hábil, diagnóstico e tratamento do câncer invasivo, além de cuidado paliativo [World Health Organization (WHO), 2014].

Um programa abrangente inclui três componentes interdependentes: primário, secundário e terciário. O objetivo 


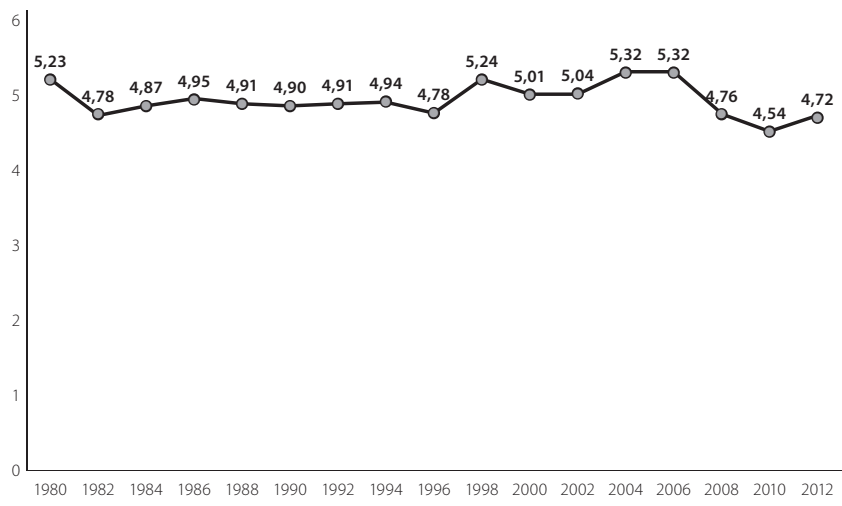

Fonte: Ministério da Saúde (MS)/Secretaria de Vigilância em Saúde (SVS)/Departamento de Análise de Situação de Saúde (Dasis)/Coordenação Geral de Informações e Análise Epidemiológica (CGIAE)/Sistema de Informação sobre Mortalidade (SIM); Ministério do Planejamento, Orçamento e Gestão (MP)/Fundação Instituto Brasileiro de Geografia e Estatística (IBGE); MS/Instituto Nacional de Câncer (Inca)/Coordenação de Prevenção e Vigilância (Conprev)/Divisão de Vigilância.

Figura 6. Taxas de mortalidade por câncer de colo do útero, ajustada por anos, segundo localização primária do tumor, por 100 mil mulheres, pela população brasileira de 2010, Brasil, com idade de 18 a 99+, entre 1980 e 2013 [Ministério da Saúde (Brasil). Instituto Nacional de Câncer (Inca). Organização PanAmericana da Saúde, 2014].

do componente primário é reduzir o número de casos de infecção por HPV por meio de intervenções como: vacinação de meninas entre 9 e 13 anos, antes do início da vida sexual; educação sexual para meninos e meninas com o objetivo de reduzir a transmissão do HPV [assim como outras doenças sexualmente transmissíveis (DST)]; promoção do uso do preservativo masculino. O componente secundário visa diminuir a incidência e a prevalência do CCU e a mortalidade associada. Por fim, o componente terciário almeja diminuir o número de óbitos devidos ao CCU, por meio da facilitação do diagnóstico e tratamento da doença, além de cuidados paliativos que visem ao alívio da dor e do sofrimento das pacientes [World Health Organization (WHO), 2014].

Para a OMS, o tratamento deve ser iniciado após o diagnóstico definitivo do CCU, a partir do exame histopatológico de uma biópsia. As opções de tratamento incluem intervenção cirúrgica, radioterapia e quimioterapia, e podem ser realizadas de forma combinada. Sob a perspectiva da OMS, uma vez que a paciente tenha sido informada e aconselhada sobre as características da doença e os tratamentos disponíveis, cabe a ela a decisão de escolher o tratamento de sua preferência (salvo casos especiais que requeiram tratamento específico) e quando iniciá-lo [World Health Organization (WHO), 2014].

\section{Prevenção, políticas de saúde da mulher e CCU}

Nos Estados Unidos (EUA), o câncer cervical invasivo apresentou queda vertiginosa na taxa de incidência desde que o rastreio citológico, realizado por meio do teste de Papanicolau, foi introduzido, na década de 1950. Essa queda ocorreu apesar do aumento de fatores de risco para câncer cervical, tais como início da relação sexual em idade mais jovem, mais parceiros sexuais e maior prevalência de infecção por HPV e de tabagismo. O sucesso na prevenção reflete três fatores: (1) a progressão da displasia de baixo grau para uma displasia mais grave, carcinoma in situ e câncer invasivo é geralmente lenta, havendo tempo para a detecção; (2) anormalidades celulares associadas podem ser identificadas; (3) há disponibilidade de um tratamento eficaz para lesões pré-malignas. Consequentemente, o carcinoma de células escamosas invasivo do colo uterino é uma doença altamente evitável (Agency for Healthcare Research and Quality, 2002).

A introdução de programas de rastreamento na população naìve para triagem reduz taxas de CCU em 60\% a 90\% no prazo de três anos após a implementação. Essa redução da mortalidade e morbidade com a introdução de triagem com o teste de Papanicolau é consistente entre populações. Como resultado, esse teste é uma das intervenções preventivas com recomendação "A" da US Preventive Services Task Force (Agency for Healthcare Research and Quality, 2002).

Nos EUA, a incidência de CCU diminuiu de 14,2 novos casos por 100 mil mulheres em 1973 para 7,8 por 100 mil em 1994. Contudo, ele continua a ser a nona causa de mortes por câncer mais comum. Dos objetivos de prevenção do cânceres estabelecidos em Healthy People 2000, que incluíram o câncer colorretal, de pulmão e de mama, as taxas de mortalidade do CCU foram as mais distantes do alvo na revisão intermediária dos dados. A meta era a redução da mortalidade para 1,3 morte por 100 mil mulheres, porém a taxa em 2002 foi de 2,7 mortes por 100 mil, apenas ligeiramente abaixo dos 2,8 por 100 mil em 1987 (Agency for Healthcare Research and Quality, 2002).

O National Breast and Cervical Cancer Early Detection Program (NBCCEDP) é um programa americano que tem por finalidade auxiliar mulheres que se encontram em situação econômica vulnerável a ter acesso aos serviços de rastreamento para detecção precoce do câncer de mama e do CCU. Esse programa é direcionado para mulheres de baixa renda, sem plano de saúde, entre 18 e 64 anos, e visa fornecer exames clínicos da mama, mamografias e testes de Papanicolau para as pacientes elegíveis. Desde a sua implantação em 1991 até 2002, o NBCCEDP atendeu aproximadamente 1,75 milhão de mulheres, que puderam realizar 2.038.118 mamografias e 2.305.936 testes de Papanicolau. Graças a tais exames de rastreamento, 9.956 casos de câncer de mama, 12.187 casos de lesões cervicais pré-cancerosas e 832 casos de CCU invasivo foram diagnosticados (Figura 7) (Centers for Disease Control and Prevention, 2003).

Na Inglaterra, o objetivo do NHS Cervical Screening Programme, disponível para pacientes entre 25 e 64 anos (Public 


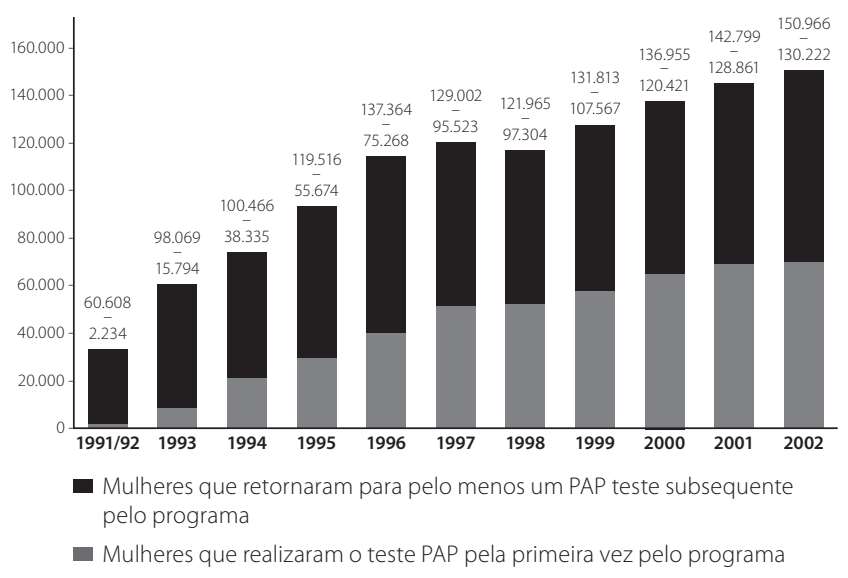

PAP: teste de Papanicolau.

* Durante esse período, 1.329.523 mulheres no mínimo realizaram teste de Papanicolau por meio do National Breast and Cervical Cancer Early Detection Program (NBCCEDP).

Figura 7. Número de mulheres que realizaram o teste de Papanicolau por meio do NBCCEDP, entre 1991 e 2002*. Adaptada: de Center for Disease Control and Prevention, 2003.

Health England, 2015), é reduzir o número de mulheres com potencial para desenvolver CCU e sua taxa de mortalidade. Desde a sua implantação, em 1988, o número de casos de CCU diminuiu em 7\% ao ano (NHS Choices, 2015).

As taxas de incidência de CCU na Grã-Bretanha diminuíram, de forma geral, desde o final da década de 1970. As taxas de incidência na Europa padronizadas por idade atingiram o pico em 1985 a 1987, diminuindo em 50\% para o seu ponto mais baixo entre 2004 e 2006, e desde então mantiveram-se estáveis. A diminuição dramática nas taxas desde o final dos anos 1980 segue a introdução do NHS Cervical Screening Programme no Reino Unido em 1988. As maiores reduções foram em mulheres com idade de 50 a 64 e 65 a 79, com taxas de incidência padronizadas por idade diminuindo em 63\% e 66\%, respectivamente, em 1985 a 1987 e 2010 a 2012 (Cancer Research UK, n.d.-a).
As taxas de mortalidade por CCU na Europa, padronizadas por idade, diminuíram 81\% entre 1971 e 2012. Os valores das taxas de mortalidade caíram de forma constante ao longo dos anos 1970 e 1980 (caindo 22\% entre 1971 e 1989) e, então, assim como as taxas de incidência, apresentaram queda ainda mais acelerada após melhorias no programa de rastreamento (diminuição de 43\% entre 1987 e 1999). Estima-se que o rastreio do CCU evite cerca de 5 mil mortes por ano no Reino Unido. Ao longo da última década (entre 2001 e 2012), as taxas de mortalidade padronizadas por idade na Europa diminuíram 21\%. As taxas entre mulheres de 65 a 79 anos de idade permaneceram estáveis entre os anos 1970 e início de 1980, diminuindo em seguida. Já para mulheres com idade entre 25 e 49 anos, as taxas de mortalidade aumentaram entre meados de 1970 e 1980, contudo apresentaram posterior redução (Cancer Research UK, n.d.-b).

No Brasil, políticas públicas voltadas para o CCU foram desenvolvidas a partir da década de 1970 (Figura 8) [Ministério da Saúde (Brasil). Instituto Nacional de Câncer (Inca), 2014].

\section{Diagnóstico}

Para o diagnóstico, o Inca recomenda exame ginecológico, citopatologia, colposcopia e biópsia em caso de citopatologias anormais de alto risco (lesões exofíticas e endofíticas), e exame histopatológico do material biopsiado [Ministério da Saúde (Brasil). Instituto Nacional do Câncer, 2000]. De acordo com o Projeto Diretrizes [Federação Brasileira das Sociedades de Ginecologia e Obstetrícia (Febrasgo) \& Sociedade Brasileira de Cancerologia, 2001], o rastreamento por meio de exame citológico com técnica de Papanicolau é indicado para todas as mulheres sexualmente ativas independentemente da idade. As mulheres que não tiveram atividade sexual estão fora de risco para CCU e não precisam de rastreamento. Se os exames forem normais, a coleta de citologia pode ser interrompida aos 65 anos. O tempo entre as coletas de citologia deve variar entre 1 e 3 anos, dependendo dos fatores de risco. Na presença de algum fator como nível socioeconômico baixo, início precoce

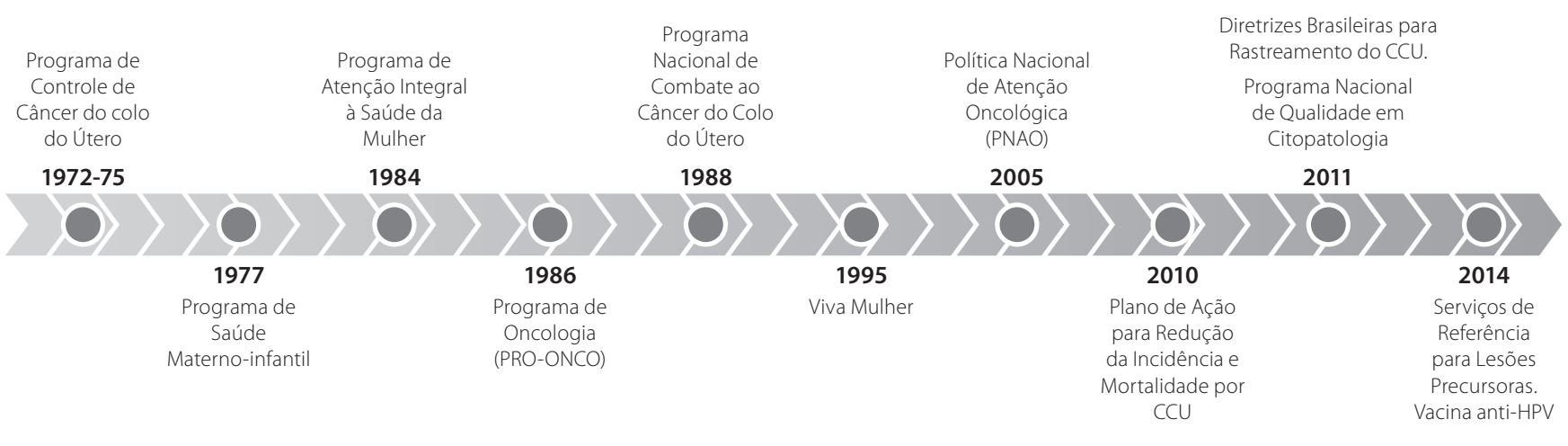

Figura 8. Linha do tempo das políticas públicas para CCU, Brasil [Ministério da Saúde (Brasil). Instituto Nacional de Câncer (Inca), 2011]. 
da atividade sexual e história de múltiplos parceiros, as coletas citológicas devem ser feitas anualmente [Federação Brasileira das Sociedades de Ginecologia e Obstetrícia (FEBRASGO) \& Sociedade Brasileira de Cancerologia, 2001].

Os resultados falso-negativos da citologia cervical variam de 1,55\% a 55\%. Para obtenção de células endocervicais, a combinação mais eficiente é o uso da escova para coleta endocervical e uma espátula do tipo longa. O diagnóstico de lesões pré-neoplásicas e microinvasoras é sugerido pela citologia tríplice e deve ser confirmado por colposcopia e biópsia dirigida. Quando a colposcopia for insatisfatória ou os resultados da citologia e da biópsia dirigida não forem compatíveis, a conização é indicada como método de diagnóstico. Os casos diagnosticados pela citologia, assim como as pacientes com lesões visíveis do colo, devem ser encaminhados para a colposcopia [Federação Brasileira das Sociedades de Ginecologia e Obstetrícia (Febrasgo) \& Sociedade Brasileira de Cancerologia, 2001].

Devido ao seu fácil diagnóstico em estágios precoces por meio da técnica de Papanicolau, houve uma importante redução da morbimortalidade por essa patologia ao longo das últimas décadas, nos países que implementaram massivamente programas de rastreamento e tratamento (Katz \& Wright, 2006; Schiffman \& Castle, 2005).

No Brasil, apesar do avanço nas políticas públicas para o controle do CCU, a maioria dos diagnósticos ainda é feita em fases avançadas da doença. Um grande estudo transversal foi realizado em três centros nacionais especializados, com análise de dados do período de 2000 a 2009, incluindo um total de 37.638 casos de CCU, e concluiu que $70,6 \%$ das muIheres foram diagnosticadas em estádio avançado ( $\geq$ II B) da doença (Thuler et al., 2014). Esse padrão se repetiu em todas as faixas etárias avaliadas. Foram considerados preditores independentes de diagnóstico em estádio avançado no Brasil: tipo histológico carcinoma de células escamosas, idade $\geq 50$ anos, vivendo sem companheiro, da raça ou cor da pele negra e com baixa escolaridade.

Outros estudos nacionais comprovam esses achados, como análise feita no Inca, de 1999-2004, incluindo 4.950 muIheres com CCU, que encontrou 72,1\% de neoplasia invasora, sendo 68,4\% em fase localmente avançada (estádios II e III), e apenas 27,9\% de estádio I (Calazan et al., 2008).

\section{Tratamento}

No âmbito do Sistema Único de Saúde do Brasil, o Protocolo Clínico e Diretrizes Terapêuticas (PCDT) em Oncologia do Ministério da Saúde mais recente, publicado em 2014, não define nenhuma estratégia de tratamento para o CCU [Ministério da Saúde (Brasil). Secretaria de Atenção à Saúde, 2014].

Segundo o Projeto Diretrizes [Federação Brasileira das Sociedades de Ginecologia e Obstetrícia (Febrasgo) \& Socie- dade Brasileira de Cancerologia, 2001], da Associação Médica Brasileira e Conselho Federal de Medicina, publicado em 2001, o tratamento recomendado para lesões pré-neoplásicas do CCU varia de acordo com a sua classificação, sendo: NIC I, conduta expectante ou destrutiva; NIC II, conduta destrutiva ou ablativa, e NIC III, ablação (conização ou histerectomia). Diversos fatores devem ser considerados ao determinar-se a terapêutica adequada para lesões de alto grau, como idade da paciente, condição clínica e o desejo de manter fertilidade. Lesões de alto grau devem ser tratadas pela conização, que varia de acordo com a extensão da lesão no canal endocervical, podendo ser conização clássica com bisturi, com alça diatérmica ou a laser. Ainda, para pacientes consideradas inoperáveis, a radioterapia é uma alternativa. Para adenocarcinoma in situ, a histerectomia deve ser considerada a melhor opção terapêutica. O tratamento de carcinomas invasores não localmente avançados de estádio IA2 e IB1 mais indicado é o cirúrgico exclusivo, com indicação de radioterapia (externa e intracavitária) apenas para pacientes sem condições clínicas para cirurgia, ou aquelas que, após o procedimento cirúrgico, apresentarem comprometimento ganglionar, doença parametrial residual ou margens cirúrgicas comprometidas (apenas externa). No estádio IB2, indica-se a cirurgia e linfadenectomia seguida de radioterapia se houver margens cirúrgicas comprometidas e invasão parametrial ou metástases para gânglios, radioterapia externa associada à braquiterapia, seguida ou não de cirurgia, e associação da radioterapia e da quimioterapia, seguida ou não de cirurgia. A radioterapia exclusiva ou histerectomia radical no estádio IIA tem boas taxas de sucesso. As neoplasias classificadas nos estádios IIB, IIIA, IIIB e IVA são denominadas CCU localmente avançado. O melhor esquema de tratamento para esse grupo de pacientes não está definido. As alternativas incluem: quimiossensibilização, radioterapia exclusiva, quimioterapia neoadjuvante seguida de radioterapia e cirurgia, quimioterapia neoadjuvante seguida de cirurgia e exenteração pélvica [Federação Brasileira das Sociedades de Ginecologia e Obstetrícia (Febrasgo) \& Sociedade Brasileira de Cancerologia, 2001].

O Inca recomenda para esse grupo de pacientes com CCU localmente avançado o uso de radioterapia exclusiva. A terapêutica para casos de CCU IVB é controversa, sendo a quimioterapia, a radioterapia e as cirurgias paliativas indicadas de acordo com cada caso [Ministério da Saúde (Brasil). Instituto Nacional do Câncer, 2000].

As diretrizes nacionais do Ministério da Saúde e do Projeto Diretrizes encontram-se claramente defasadas, quando é olhado o cenário internacional, tanto para publicações científicas com opções terapêuticas quanto para diretrizes de tratamento, ou mesmo o cenário nacional de diretrizes recentes e preconizadas por sociedades médicas.

A associação da terapia biológica com a quimioterápica é indicada pelo Manual de Oncologia Clínica de 2016 (Cen- 
tro Oncológico Antônio Ermínio de Moraes. 12a edição. Manual de Oncologia Clínica do Brasil. 2016. n.d.) para a primeira escolha de tratamento das portadoras de CCU em estádio avançado. Essa recomendação baseia-se nas evidências de diversos estudos clínicos que mostram maior eficácia e segurança da terapia com biológico quando comparada à quimioterapia padrão. A maioria dos agentes comumente utilizados, tais como paclitaxel, irinotecano, gencitabina e pemetrexede, apresenta uma atividade mínima no tratamento da doença persistente ou recorrente em uma área previamente irradiada (McGuire et al., 1996; Miller et al., 2008; Morris et al., 1998; Schilder et al., 2000).

Diversos estudos suportam a eficácia e a segurança da terapia com medicamento biológico para o CCU avançado. Um grande estudo de fase III comparou o padrão de tratamento quimioterápico com a associação de um biológico (bevacizumabe) e demonstrou ganho significativo na sobrevida global. Em pacientes diagnosticadas em estádio avançado da doença, a mediana de sobrevida global passou de 13,3 meses, quando tratadas somente com quimioterapia, para 17 meses, quando houve a associação com biológico (Tewari et al., 2014). Além da melhora na sobrevida global e na sobrevida livre de progressão, a incorporação de bevacizumabe ao tratamento não foi acompanhada de nenhuma deterioração na qualidade de vida relacionada à saúde (Penson et al., 2015).

A defasagem de protocolos na saúde pública torna-se mais relevante, uma vez que a maioria das pacientes é diagnosticada em fases tardias da doença e necessita de tratamentos para tais estádios. A Figura 9 apresenta a evolução do tratamento inicial e do tratamento avançado com quimioterapia no SUS para CCU, ao longo dos anos. Observa-se, em 2008-2009, um crescimento do tratamento inicial, com queda a partir de 2009. Já o tratamento para fases avança-

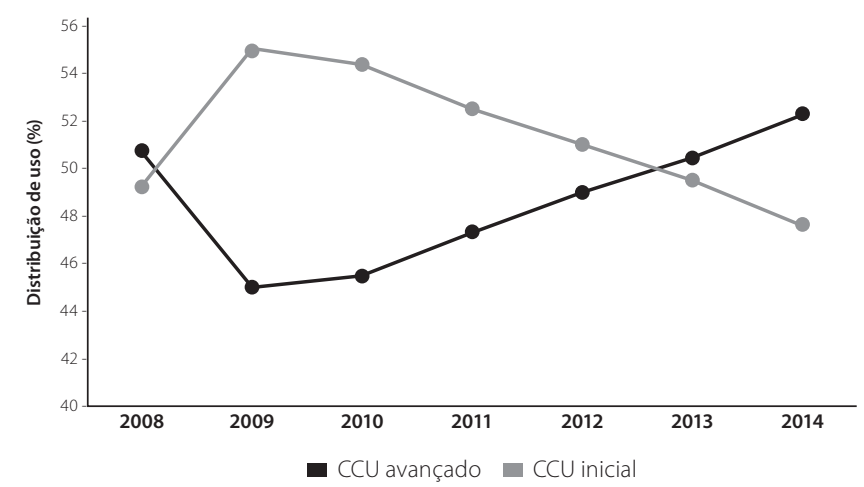

CCU inicial: 0304040045 quimioterapia do carcinoma epidermoide/adenocarcinoma do colo uterino; CCU avançado: 0304020184 quimioterapia do carcinoma epidermoide/adenocarcinoma do colo ou do corpo uterino avançado.

Figura 9. Evolução do tratamento para câncer de colo do útero inicial versus avançado, Brasil, TabNet, 2008-2014 [Ministério da Saúde (Brasil). Departamento de Informática do SUS (Datasus), 2016a, 2016b] das entra em franco crescimento a partir de 2009. Em relação à distribuição de acordo com a faixa etária, observa-se uma tendência de aumento do tratamento em pacientes acima de 55 anos de idade (Figura 10) [Ministério da Saúde (Brasil). Departamento de Informática do SUS (Datasus), 2016a, 2016b].

\section{Cenário brasileiro - necessidades não atendidas e perspectivas}

O Ministério da Saúde elaborou o Plano de Ações Estratégicas para o Enfrentamento das Doenças Crônicas Não Transmissíveis (DCNT) no Brasil, 2011-2022. Entre as metas propostas, estão a ampliação da cobertura de mamografia para mulheres entre 50 e 69 anos, o aumento da cobertura de exame citopatológico para mulheres de 25 a 64 anos e o tratamento de todas as mulheres com diagnóstico de lesões precursoras de câncer. As principais ações para o combate ao CCU e da mama baseiam-se no aperfeiçoamento do rastreamento dessas doenças, na universalização desses exames a todas as mulheres e na garantia de acesso total ao tratamento de lesões precursoras de câncer [Ministério da Saúde (Brasil). Secretaria de Atenção à Saúde, 2013]. Entre essas ações, encontram-se as Linhas de Cuidado, que estabelecem o percurso do paciente durante a assistência, estruturado em critérios epidemiológicos e de regionalização, com objetivo de alcançar bons resultados clínicos, a custos compatíveis e com base nas evidências científicas. A Linha de Cuidado do Câncer do Colo do Útero tem como objetivo garantir o acesso humanizado e integral às ações e aos serviços qualificados. É baseada nas diretrizes de prevenção e detecção precoce, no Programa Nacional de Qualidade da Citologia, no acesso à confirmação diagnóstica e no tratamento adequado em

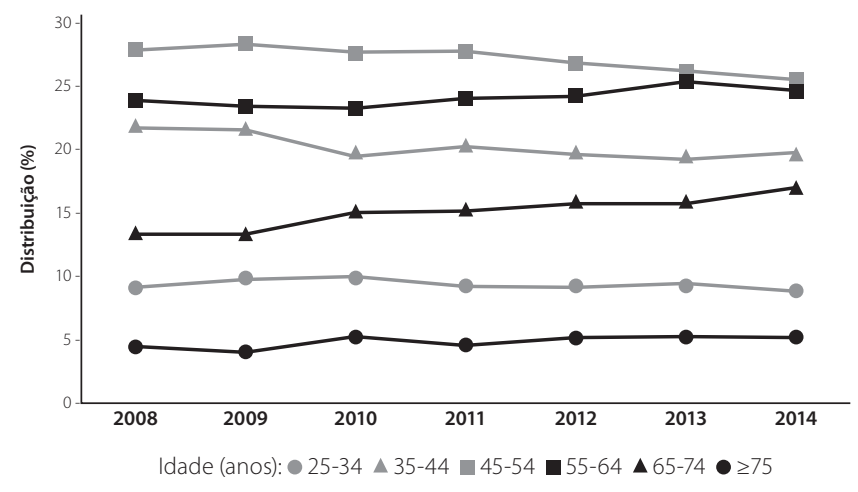

Os procedimentos considerados foram: 0304040045 quimioterapia do carcinoma epidermoide/adenocarcinoma do colo uterino e 0304020184 quimioterapia do carcinoma epidermoide/adenocarcinoma do colo ou do corpo uterino avançado.

Figura 10. Evolução do tratamento por faixa etária, Brasil, TabNet, 2008-2014 [Ministério da Saúde (Brasil). Departamento de Informática do SUS (Datasus), 2016a, 2016b]. 
tempo oportuno [Ministério da Saúde (Brasil). Secretaria de Atenção à Saúde, 2013].

Apesar das metas propostas pelo Plano de Ações Estratégicas para o Enfretamento das DCNT, a comparação de dados de 2010 e 2013 mostra apenas um aumento discreto da taxa de cobertura do exame Papanicolau (de 82,2\% para 82,9\%). Segundo o Vigitel, a meta manteve-se estável nesse período, porque a cobertura existente já era elevada (Malta \& Silva Jr., 2014). Uma nova análise realizada para o período de 2011 a 2015 também mostrou estabilidade na realização da citologia oncótica para rastreamento do CCU (Malta et al., 2016). A partir da análise do cenário nacional de elaboração de políticas de saúde voltadas para o CCU e a evolução temporal da epidemiologia da doença, algumas observações podem ser feitas.

É sabido que o CCU possui bom prognóstico quando diagnosticado em fases precoces, e diversos programas foram desenvolvidos com esse foco. Esses programas conseguiram alcançar um aumento do acesso da população ao teste de Papanicolau, porém, em análise dos últimos cinco anos, o patamar de cobertura se manteve estável (Malta et al., 2016). Embora a taxa de cobertura esteja estável, ela é considerada alta, em torno de 83\% (Malta \& Silva Jr., 2014). Apesar disso, pesquisas apontam que a grande maioria da população brasileira (mais de 70\%) ainda continua sendo diagnosticada em fases avançadas da doença (Calazan et al., 2008; Thuler et al., 2014), o que impacta diretamente e de forma negativa no prognóstico.

Enquanto na Europa, entre 2001 e 2012, foi observada queda de 21\% na taxa de mortalidade (Cancer Research UK, n.d.-b), as metas de queda na taxa de mortalidade por CCU no Brasil não foram atingidas e, nas últimas três décadas, esses valores se mantiveram constantes, o que provavelmente tem no diagnóstico tardio uma de suas principais causas [Ministério da Saúde (Brasil). Instituto Nacional de Câncer (Inca). Organização Pan-Americana da Saúde, 2014]. Soma-se a isso a defasagem nos protocolos de tratamento voltados para a saúde pública no Brasil, especialmente para o segmento de população com doença avançada [Ministério da Saúde (Brasil). Secretaria de Atenção à Saúde, 2014].

As metas de diagnóstico precoce e queda na taxa de mortalidade não alcançadas se refletem em necessidades médicas não atendidas e faz-se necessária uma análise criteriosa das possíveis causas associadas. A identificação do perfil de mulheres com doença avançada pode levar a reformulações em programas com foco específico para esse perfil que, provavelmente não está sendo atingido ou sensibilizado por campanhas atuais. A atualização dos protocolos de tratamento também é de fundamental importância para que sejam equiparados com diretrizes internacionais ou nacionais de sociedades médicas, que incluem tratamentos com comprovado ganho de sobrevida global.
A melhoria do acesso aos serviços de saúde e à informação é fundamental para o controle do CCU. Os serviços de saúde em todos os níveis de atendimento devem promover amplo acesso da população à informação [Ministério da Saúde (Brasil). Secretaria de Atenção à Saúde, 2013].

No Brasil, os altos índices de incidência e mortalidade por CCU evidenciam a importância da elaboração e da implementação de políticas públicas na atenção básica, voltadas à atenção integral à saúde da mulher, garantindo ações relativas ao controle dessa doença. Apesar dos avanços na difusão de medidas preventivas, o CCU continua a ser um problema de saúde importante no país. Os gestores e profissionais de saúde devem ser responsáveis pela realização dessas ações e devem possibilitar a integralidade do cuidado, unindo as ações de detecção precoce com a garantia de acesso a procedimentos diagnósticos e terapêuticos em tempo oportuno e com qualidade. A atenção básica qualificada e organizada é fundamental para o controle desses tipos de cânceres [Ministério da Saúde (Brasil). Secretaria de Atenção à Saúde, 2013].

\section{Referências bibliográficas}

Agency for Healthcare Research and Quality. Screening for cervical cancer. 2002.

Calazan CL, Raggio R, Ferreira I. O diagnóstico do câncer do colo uterino invasor em um Centro de Referência Brasileiro: tendência temporal e potenciais fatores relacionados. Rev Bras Cancerol. 2008;54(4):325-31.

Cancer Research UK [Internet]. Cervical cancer incidence statistics. n.d.-a. Disponível em: <http://www.cancerresearchuk.org/health-professional/ cervical-cancer-incidence-statistics\#heading-Two>.

Cancer Research UK [Internet]. Cervical cancer mortality statistics. n.d.-b. Disponível em: <http://www.cancerresearchuk.org/health-professional/ cervical-cancer-mortality-statistics\#heading-Two>.

Centers for Disease Control and Prevention. 1991-2002 National Report: Summarizing the First 12 Years of Partnerships and Progress Against Breast and Cervical Cancer. 2003.

Centro Oncológico Antônio Ermínio de Moraes. Manual de Oncologia Clínica do Brasil. 12a ed. São Paulo; 2016.

Federação Brasileira das Sociedades de Ginecologia e Obstetrícia (Febrasgo), Sociedade Brasileira de Cancerologia. Projeto Diretrizes: Rastreamento, diagnóstico e tratamento do carcinoma do colo do útero. 2001.

Ferlay J, Soerjomataram I, Dikshit R, Eser S, Mathers C, Rebelo M, et al. Cancer incidence and mortality worldwide: sources, methods and major patterns in Globocan 2012. Int J Cancer. 2015;136(5):E359-86.

Harper DM, Vierthaler SL. Next Generation Cancer Protection: The Bivalent HPV Vaccine for Females. ISRN Obstet Gynecol. 2011;2011:457204.

Katz I, Wright A. Preventing cervical cancer in the developing world. N Engl J Med. 2006;354(3):1110.

Malta DC, Oliveira TP, Santos MAS, Andrade SSCA, Silva MMA. Avanços do Plano de Ações Estratégicas para o Enfrentamento das Doenças Crônicas não Transmissíveis no Brasil, 2011-2015. Epidemiologia e Serviços de Saúde. 2016;25(2):373-90.

Malta DC, Silva Jr. JB. Plano de Ações Estratégicas para o Enfrentamento das Doenças Crônicas Não Transmissíveis no Brasil após três anos de implantação, 2011-2013. Epidemiologia e Serviços de Saúde. 2014;23(3):389-98. 
McGuire WP, Blessing JA, Moore D, Lentz SS, Photopulos G. Paclitaxel has moderate activity in squamous cervix cancer. A Gynecologic Oncology Group study. J Clin Oncol. 1996;14(3):792-5.

Miller DS, Blessing JA, Bodurka DC, Bonebrake AJ, Schorge JO. Evaluation of pemetrexed (Alimta, LY231514) as second line chemotherapy in persistent or recurrent carcinoma of the cervix: a Phase II study of the Gynecologic Oncology Group. Gynecol Oncol. 2008;110(1):65-70.

Ministério da Saúde (Brasil). Departamento de Informática do SUS (Datasus) [Internet]. TabNet: Procedimento 0304040045 Quimioterapia do carcinoma epidermoide/adenocarcinoma do colo uterino 2008-2014. 2016a. Disponível em: <http://tabnet.datasus.gov.br/cgi/deftohtm. exe?sia/cnv/qauf.def>.

Ministério da Saúde (Brasil). Departamento de Informática do SUS (Datasus) [Internet]. TabNet: Procedimento 0304020184 Quimioterapia do carcinoma epidermoide/adenocarcinoma do colo ou do corpo uterino avançado 2008-2014. 2016b. Disponível em: <http://tabnet.datasus.gov. $\mathrm{br/cgi/deftohtm.exe?sia/cnv/qauf.def>.}$

Ministério da Saúde (Brasil). Departamento de Informática do SUS (Datasus) [Internet]. TabNet Win32 3.0: Mortalidade - Brasil. 2013. Disponível em: <http://tabnet.datasus.gov.br/cgi/deftohtm.exe?sim/cnv/obt10uf.def>.

Ministério da Saúde (Brasil). Instituto Nacional do Câncer (Inca). Condutas do Inca: câncer do colo do útero. Rev Bras Cancerol. 2000;46(4):351-4.

Ministério da Saúde (Brasil). Instituto Nacional de Câncer (Inca). Diretrizes brasileiras para o rastreamento do câncer do colo do útero. Rio de Janeiro: Inca; 2011.

Ministério da Saúde (Brasil). Instituto Nacional de Câncer (Inca). Organização Pan-Americana da Saúde [Internet]. Atlas On-line de Mortalidade. 2014. Disponível em: <https://mortalidade.inca.gov.br/ MortalidadeWeb/>.

Ministério da Saúde (Brasil). Instituto Nacional de Câncer José Alencar Gomes da Silva (Inca) [Internet]. Controle do câncer do colo do útero - Histórico das ações. 2015. Disponível em: <http://www2.inca.gov.br/wps/wcm/ connect/acoes_programas/site/home/nobrasil/programa_nacional_ controle_cancer_colo_uteros.

Ministério da Saúde (Brasil). Instituto Nacional de Câncer José Alencar Gomes da Silva (Inca). Estimativa 2014: incidência de câncer no Brasil. Brasília: Inca; 2014.

Ministério da Saúde (Brasil). Instituto Nacional de Câncer José Alencar Gomes da Silva (Inca). Estimativa 2016: incidência de câncer no Brasil. Rio de Janeiro: Inca; 2016.

Ministério da Saúde (Brasil) [Internet]. Meninos poderão ser vacinados contra HPV a partir de 2017 - Portal Saúde. 2016. Disponível em: <http://www. brasil.gov.br/saude/2016/10/meninos-poderao-ser-vacinados-contrahpv-a-partir-de-2017>.

Ministério da Saúde (Brasil). Secretaria de Atenção à Saúde. Cadernos de atenção básica: controles dos cânceres do colo do útero e da mama. 2a ed. Brasília: Ministério da Saúde; 2013.

Ministério da Saúde (Brasil). Secretaria de Atenção à Saúde. Protocolos Clínicos e Diretrizes Terapêuticas em Oncologia. Brasília: Ministério da Saúde; 2014.
Morris M, Brader KR, Levenback C, Burke TW, Atkinson EN, Scott WR, et al. Phase II study of vinorelbine in advanced and recurrent squamous cell carcinoma of the cervix. J Clin Oncol. 1998;16(3):1094-8.

NHS Choices [Internet]. Cervical screening. 2015. Disponível em: <http://www. nhs.uk/Conditions/Cervical-screening-test/Pages/Introduction.aspx>.

Nogueira-Rodrigues A, Ferreira CG, Bergmann A, Aguiar SS, Thuler LCS. Comparison of adenocarcinoma (ACA) and squamous cell carcinoma (SCC) of the uterine cervix in a sub-optimally screened cohort: a population-based epidemiologic study of 51,842 women in Brazil. Gynecol Oncol. 2014;135(2):292-6.

Parkin DM, Whelan SL, Ferlay J, Raymond L, Young J, editors. Cancer Incidence in Five Continents, v. VII. IARC Scientific Publications n. 143. Lyon: IARC; 1997.

Pecorelli S, Zigliani L, Odicino F. Revised FIGO staging for carcinoma of the vulva, cervix, and endometrium. Int J Gynecol Obstet. 2009;105(2):107-8.

Penson RT, Huang HQ, Wenzel LB, Monk BJ, Stockman S, Long HJ, et al. Bevacizumab for advanced cervical cancer: patient-reported outcomes of a randomised, phase 3 trial (NRG Oncology-Gynecologic Oncology Group protocol 240). Lancet Oncol. 2015;16(3):301-11.

Public Health England [Internet]. Population screening programmes guidance Cervical screening: programme overview. 2015. Disponível em: <https://www.gov.uk/guidance/cervical-screening-programmeoverview>.

Schiffman M, Castle PE. The promise of global cervical-cancer prevention. New Engl J Med. 2005;353(20):2101-4.

Schilder RJ, Blessing JA, Morgan M, Mangan CE, Rader JS. Evaluation of gemcitabine in patients with squamous cell carcinoma of the cervix: a Phase II study of the gynecologic oncology group. Gynecol Oncol. 2000;76(2):204-7.

Teixeira LA. From gynaecology offices to screening campaigns: a brief history of cervical cancer prevention in Brazil. Hist Cienc SaudeManguinhos. 2015;22(1):221-39.

Tewari KS, Sill MW, Long HJ, Penson RT, Huang H, Ramondetta LM, et al. Improved survival with bevacizumab in advanced cervical cancer. N Engl J Med. 2014;370(8):734-43.

Thuler LCS, Aguiar SS, Bergmann A. Determinantes do diagnóstico em estádio avançado do câncer do colo do útero no Brasil. Rev Bras Ginecol Obstet. 2014;36(6):237-43.

UK National Screening Committee. UK NSC recommendation. 2016.

World Health Organization (WHO). Comprehensive cervical cancer control: a guide to essential practice ( ${ }^{\text {nd }}$ ed.). Geneva: WHO Press; 2014.

World Health Organization (WHO). International Agency for Research on Cancer. [Internet]. CI5 X: Cancer incidence in five continents Volume X. 2013. Disponível em: <http://ci5.iarc.fr/Cl5-X/Default.aspx>.

World Health Organization (WHO) [Internet]. Globocan 2012: Estimated Cancer Incidence, Mortality and Prevalence Worldwide in 2012. 2012. Disponível em: <http://globocan.iarc.fr/Pages/age-specific_table_sel.aspx>.

World Health Organization (WHO) [Internet]. WHO Cancer Mortality Database. 2015. Disponível em: <http://www-dep.iarc.fr/WHOdb/ WHOdb.htm>. 